\title{
Magnets and a self-retractable wire for endoscopic septotomies: from concept to first-in-human use $\square$
}

\section{(ㄷ)(1)}

Authors

François Huberland ${ }^{\mathbb{E},}$ Ricardo Rio-Tinto ${ }^{2}$, Nicolas Cauche ${ }^{3}$, Sonia Dugardeyn ${ }^{4}$, Cécilia Delattre ${ }^{3}$, Charlotte Sandersen ${ }^{5}$, Laureen Rocq ${ }^{6}$, Pauline van Ouytsel ${ }^{4}$, Alain Delchambre ${ }^{1}$, Jacques Devière ${ }^{2,4}$, Daniel Blero ${ }^{4}$

Institutions

1 Bio, Electro and Mechanical Systems Department, Université Libre de Bruxelles, Brussels, Belgium

2 Champalimaud Foundation, Lisbon, Portugal

3 Brussels Medical Device Center, Brussels, Belgium

4 Department of Gastroenterology, Hepatopancreatology, and Digestive Oncology, Erasme Hospital, Université Libre de Bruxelles, Brussels, Belgium

5 Department of Clinical Sciences, Faculty of Veterinary Medicine, University of Liège, Liège, Belgium

6 Department of Pathology, Erasme Hospital, Université Libre de Bruxelles, Brussels, Belgium

submitted 15.4.2021

accepted after revision 20.7.2021

published online 19.7.2021

\section{Bibliography}

Endoscopy 2022; 54: 574-579

DOI 10.1055/a-1554-0976

ISSN 0013-726X

(C) 2021. The Author(s).

This is an open access article published by Thieme under the terms of the Creative Commons Attribution-NonDerivative-NonCommercial License, permitting copyin and reproduction so long as the original work is given appropriate credit. Contents may not be used for commercial purposes, or adapted, remixed, transformed or built upon. (https://creativecommons.org/licenses/by-nc-nd/4.0/)

Georg Thieme Verlag KG, Rüdigerstraße 14,

70469 Stuttgart, Germany

Scan this QR-Code for the author commentary.

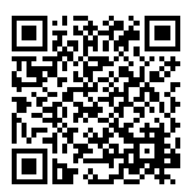

Corresponding author

Daniel Blero, MD, Hôpital Erasme, Université libre de

Bruxelles, Route de Lennik, 808-B, 1070 Bruxelles, Belgium

daniel.blero@erasme.ulb.ac.be

\section{ABSTRACT}

Background A medical device that allows simple and safe performance of an endoscopic septotomy could have several applications in the gastrointestinal (GI) tract. We have developed such a device by combining two magnets and a self-retractable wire to perform a progressive septotomy by compression of the tissues. We describe here the concept, preclinical studies, and first clinical use of the device for the treatment of symptomatic epiphrenic esophageal diverticulum (EED).

Methods The MAGUS (MAgnetic Gastrointestinal Universal Septotome) device was designed based on previous knowledge of compression anastomosis and currently unmet needs. After initial design, the feasibility of the technique was tested on artificial septa in pigs. A clinical trial was then initiated to assess the feasibility and safety of the technique.

Results Animal studies showed that the MAGUS can perform a complete septotomy at various levels of the GI tract. In two patients with a symptomatic EED, uneventful complete septotomy was observed within 28 and 39 days after the endoscopic procedure.

Conclusions This new system provides a way of performing endoluminal septotomy in a single procedure. It appears to be effective and safe for managing symptomatic EED. Further clinical applications where this type of remodeling of the GI tract could be beneficial are under investigation.

\section{Introduction}

The ability to safely and easily perform a septotomy in the gastrointestinal (Gl) tract could dramatically improve the treatment of several pathological conditions, including diverticula and post-surgical complications. Symptomatic epiphrenic esophageal diverticulum (EED) is an example of such a condition, where the current standard surgical treatment is associated with high rates of morbidity $(0 \%-75 \%)$ and mortality $(0 \%-$ $11.1 \%$ ) [1] and the latest therapeutic approach (diverticular peroral endoscopic myotomy [D-POEM]) is technically demanding and preferred only for motility disorder-related EED [2]. 
In 2015, we proposed an alternative method to treat EED, consisting of the use of a pair of magnets to induce a compression anastomosis between the diverticulum and the esophagus. The compression induces ischemia, leading to necrosis, inflammation, and ultimately to a healing process, allowing the creation of an endoluminal anastomosis. During revisional endoscopy, the residual septum is cut using an electrical knife [3]. The feasibility of this approach was confirmed by another team [4]. Although effective, this technique requires two interventions, the second one being technically challenging.

Based on this previous experience, we have developed a new device that provides marsupialization of an EED, called the MAGUS (MAgnetic Gastrointestinal Universal Septotome). It consists of two magnets that are placed on each side of the septum of the diverticulum, which are linked by a self-retractable suture wire ( Fig. 1). By applying a constant pressure with both, the magnets induce a compression anastomosis and the wire induces what we call "wire compression cutting" (WCC). After confirming the method in proof-of-concept experiments in a pig model, we initiated a prospective human study. We present here the results of this study in terms of conceptual design, technical preclinical success, and early clinical outcomes of the first two patients with an EED enrolled in the study.

\section{Methods}

\section{Magnet and wire septotome}

The MAGUS device (BMDC, Brussels, Belgium) consists of three parts ( $\triangleright$ Fig. 2a): a single magnet $(18-\mathrm{mm}$ wide and $4.2-\mathrm{mm}$ thick); the self-retractable wire (a $0.4-\mathrm{mm}$ braided non-resorbable surgical wire); and a magnetic box (19-mm wide and 8.3$\mathrm{mm}$ thick), which contains two magnets that encompass the spring-activated wire winding system. The device concept was patented in 2018 (EP2018/055106 - WO/2018/158395).

Acceptable ranges of force and pressure applied by the magnets (i. e. the single magnet and the magnetic box) and the wire have been calculated based on four magnetic devices found in the literature. In this paper, the WCC and magnetic compression anastomosis are considered as two similar physiological phenomena that are induced by pressure. The four magnetic devices were the IMAS [5], the Magnamosis system [6], the Flourish device [7], and magnets created by Cook (Winston Salem, North Carolina, USA) and used in different studies $[3,8]$. From pictures, the information present in these reports, and theoretical calculation using the FEMM software (www. femm.info), the minimal force needed to avoid dislodgment of the magnets is $2.2 \mathrm{~N}$ at $2 \mathrm{~mm}$ and the pressure needed to induce ischemia ranges from 6.4 to $150 \mathrm{kPa}$ at $2 \mathrm{~mm}$.

The force (pressure) of attraction between the two magnets of the MAGUS ranges from $4 \mathrm{~N}(17 \mathrm{kPa})$ to $5 \mathrm{~N}(21 \mathrm{kPa})$ at $2 \mathrm{~mm}$, while the mean pressure applied by the wire is estimated to be between 50 and $70 \mathrm{kPa}$ for a 40 -mm high septum.

Once in place, the device is expected to achieve the entire cutting process within 28 days [3,5-8].

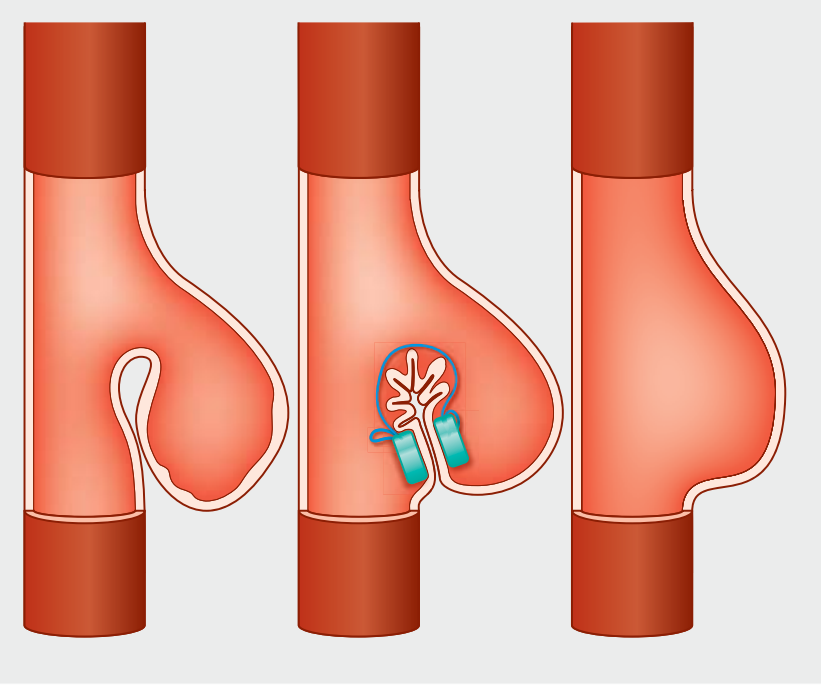

- Fig. 1 Diagram showing the system of magnets and wire that are used to cut the septum of the diverticulum, achieving a marsupialization. Both the magnets and the wire cut by applying a continuous pressure inducing pressure necrosis and wound healing with fibrosis.

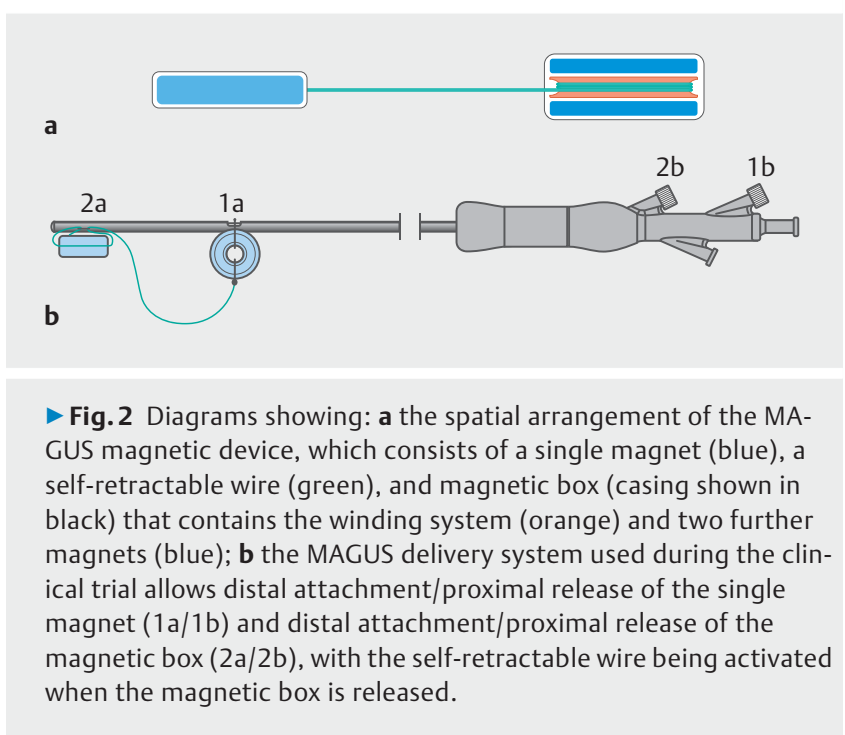

\section{Delivery procedure}

The MAGUS magnetic device is attached to a custom delivery system that keeps both magnets apart and the self-retractable wire system inactivated during the delivery procedure. The proximal and distal magnets can be released independently from one another through a dedicated handle ( $\downarrow$ Fig. $\mathbf{2 b}$ ).

The main steps of the procedure are presented in $>$ Fig. 3 . First, the delivery system is mounted on a pre-installed guidewire. Next, the endoscope is inserted alongside and a forceps is used to mobilize the proximal magnet. After delivery, it is pushed by the endoscope to the bottom of the diverticulum. The distal magnet is then pulled to meet with the proximal magnet across the septum. Once the meeting is confirmed, the distal 


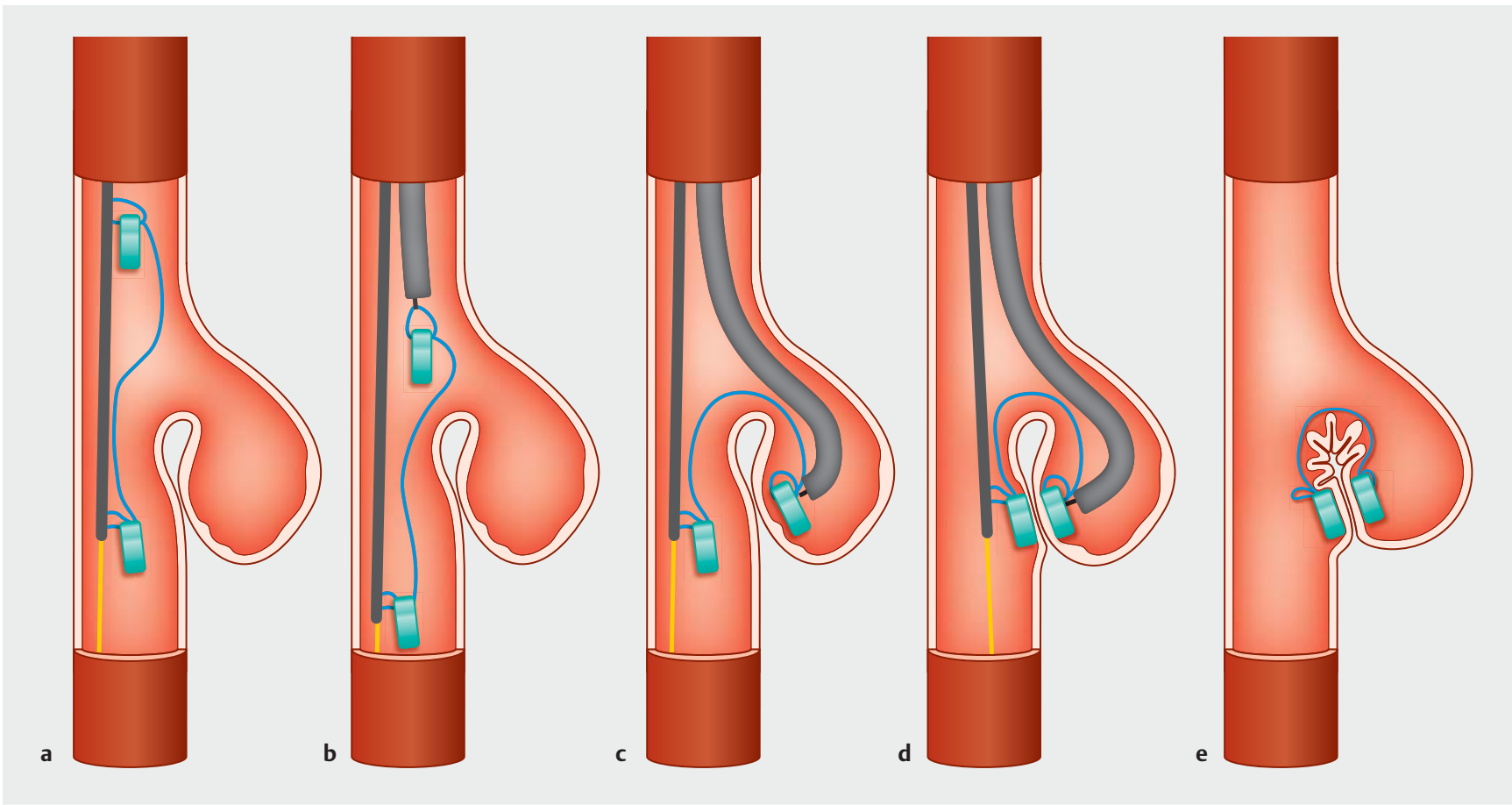

- Fig. 3 The steps in the delivery of the MAGUS system are: a insertion of the device; $\mathbf{b}$ mobilization of the proximal magnet; $\mathbf{c}$ pulling of the catheter; $\mathbf{d}$ the meeting of the magnets; $\mathbf{e}$ release of the distal magnet and activation of the self-retractable wire.

magnet is released, which also activates the self-retractable wire.

\section{Animal studies}

The feasibility of WCC was assessed in animal experiments conducted on pig models: one Yucatan (male, 1-year old, $51 \mathrm{~kg}$ ) and three Pietrain x Landrace (females, 3-4-months old, $36-42 \mathrm{~kg}$ ).

An artificial septum was created surgically in the stomach of the four different animals, resulting in a double stomach layer septum, with apposition of the two serosae. After 2 weeks, the magnets were implanted on this septum through a gastrotomy to assess the feasibility of the WCC [9].

For pig \#4, it was not possible to implant the magnets in a secure position that would avoid migration. It was therefore decided to implant them in a bowel loop, through an enterotomy. This created a septum between the afferent and efferent limbs, as illustrated in > Fig. 4. The magnets were implanted before activating the cutting wire manually.

Because magnetic compression anastomosis has been proven to work in esophageal diverticula [3], we did not consider the specific histology of the tissue when creating the artificial septa.

The animals were fed from day +1 after surgery. Follow-up fluoroscopy was performed on day +1 and day +7 . The animals were sacrificed at day 14 .

\section{Clinical trial}

A safety and feasibility clinical trial was initiated at Erasme Hospital, Université Libre de Bruxelles, Brussels in February 2020, after agreement from the ethical committee of the hospital

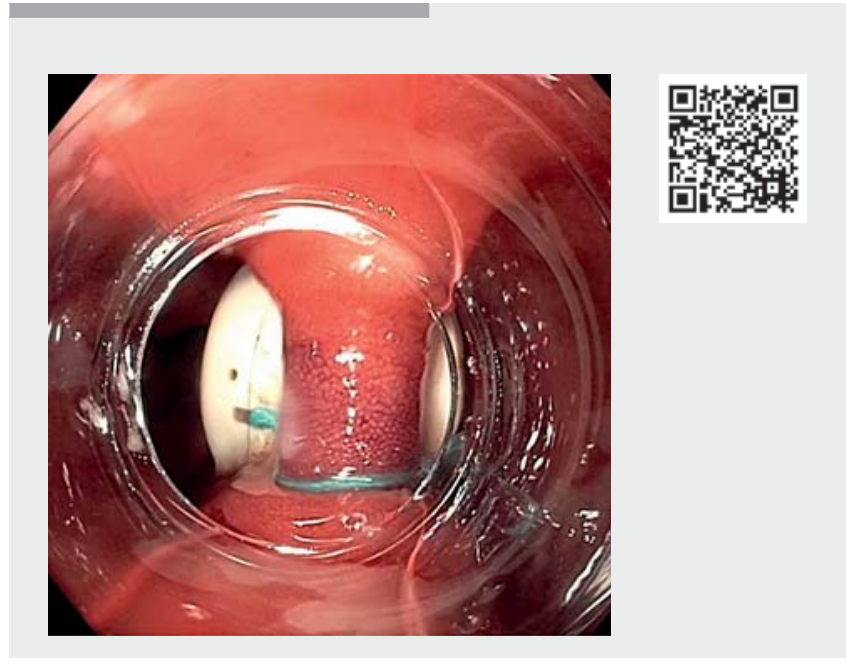

Video 1 The steps in the MAGUS delivery procedure are as follows: after the diverticulum has been identified, the proximal magnet is mobilized by the endoscope and placed at the bottom of the diverticulum; the distal magnet is then pulled back via the delivery catheter until both magnets meet at the bottom of the septum; after good positioning of the magnets has been confirmed on a fluoroscopic image, the distal magnet is released and the self-retractable wire is activated.

Online content viewable at:

https://doi.org/10.1055/a-1554-0976 

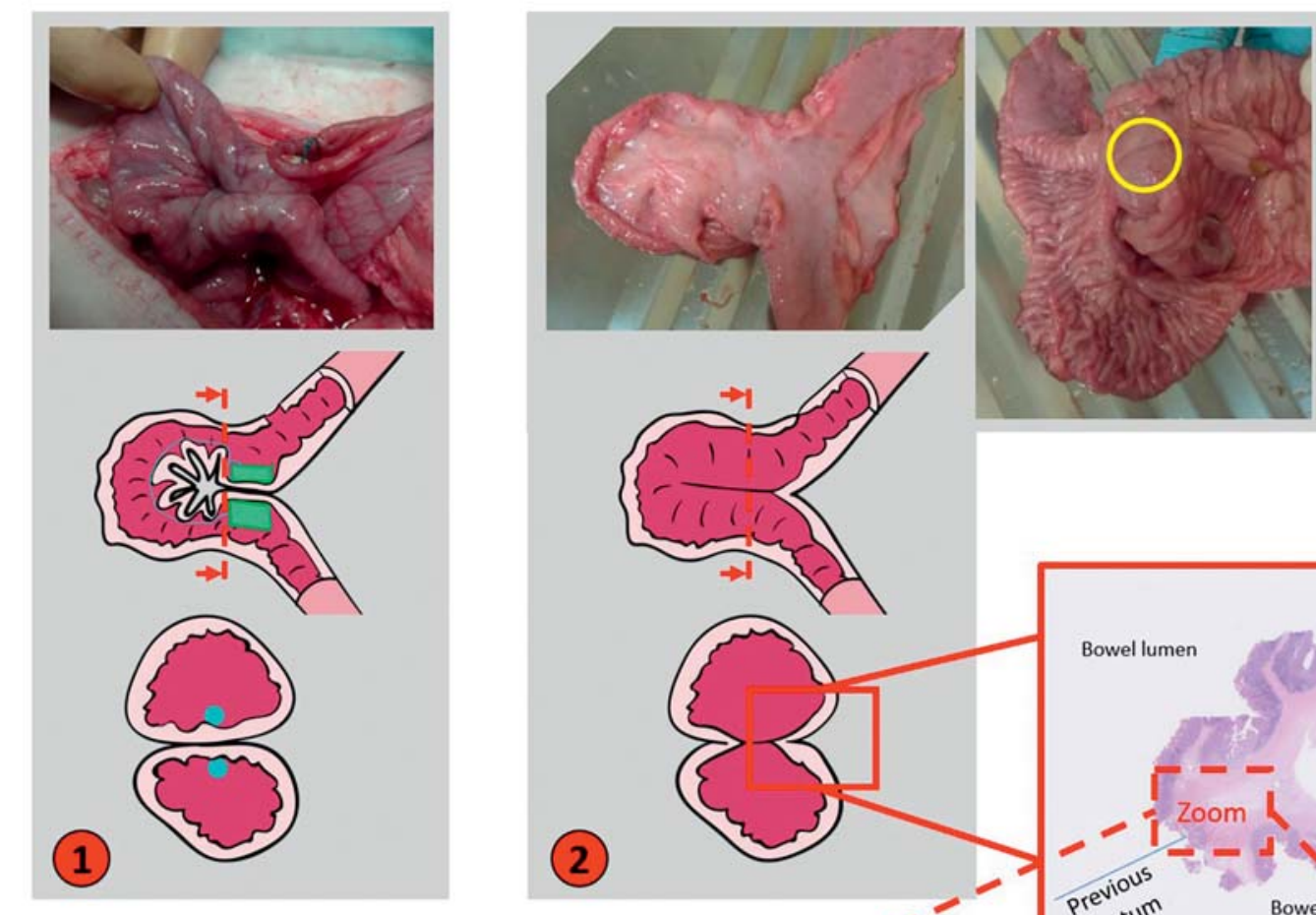

(2)
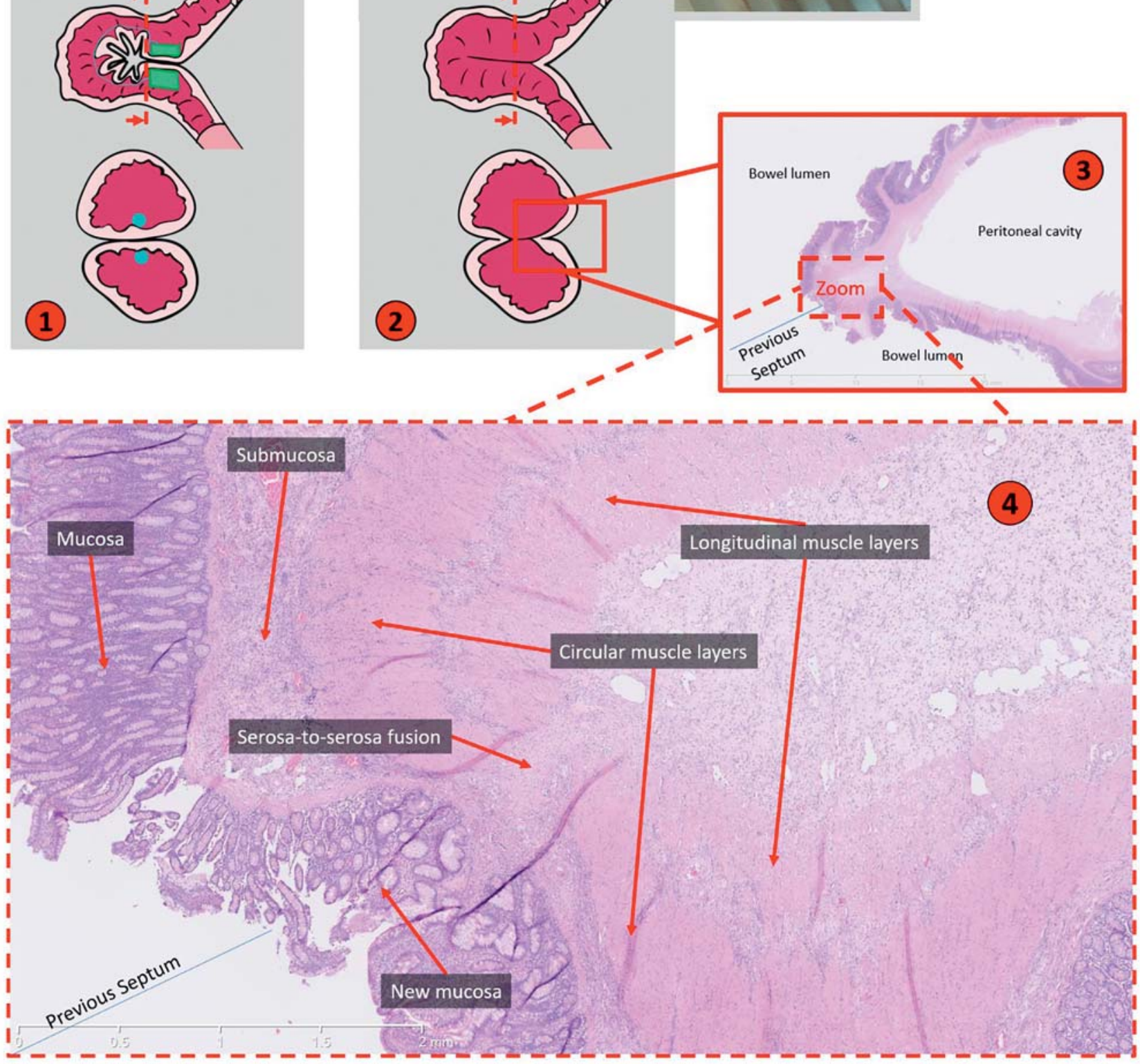

- Fig. 4 Diagrams and photographs from pig \#4 showing: a implantation of the MAGUS device (external picture, with internal and cut-view schematics); $\mathbf{b}$ the resulting cut (the yellow circle shows the cutting line); $\mathbf{c}$ macroscopic histological view; $\mathbf{d}$ microscopic histological view, with fusion of the layers and new mucosa growing on top and no significant fibrosis present. 

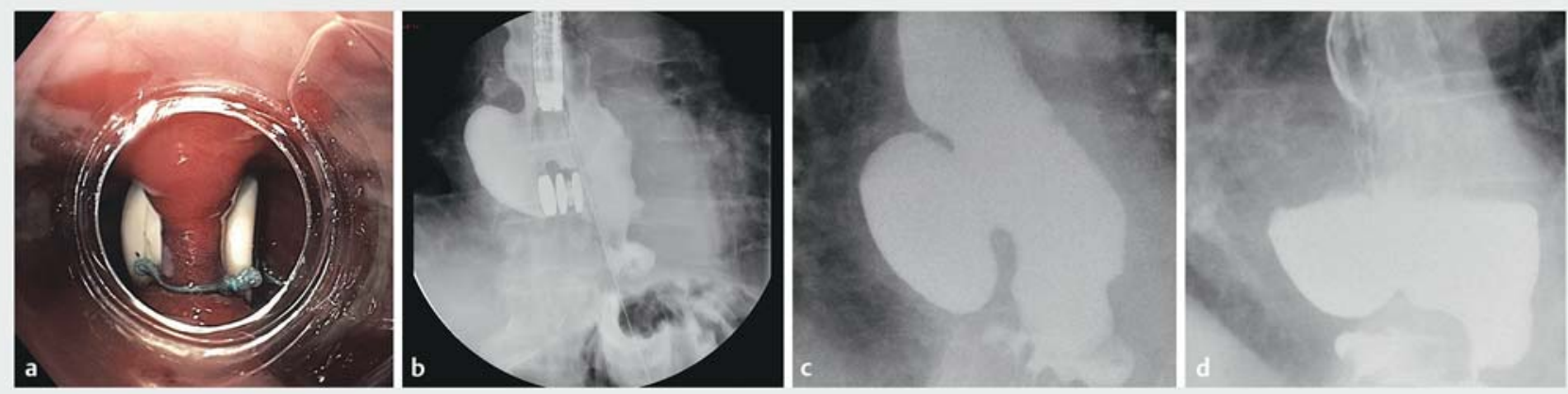

- Fig. 5 Images from the first patient showing: $\mathbf{a}, \mathbf{b}$ the implanted device: $\mathbf{a}$ at the end of the intervention endoscopic; $\mathbf{b}$ on radiological view; c,d barium swallow from: $\mathbf{c}$ baseline; $\mathbf{d} 3$ months after insertion of the MAGUS, with almost complete disappearance of the septum.

and the Belgian health authority, for patients presenting with a symptomatic EED or anatomical conditions associated with a septum.

With the patient under general anesthesia, the MAGUS was implanted as described in > Fig. 3 and shown in > Video 1. A transparent cap was used to facilitate magnet manipulation during positioning. A follow-up endoscopy was performed at 24 hours, as per protocol. Clinical and radiological follow-up was scheduled at 14,28 , and 90 days. The primary endpoints included the technical success of implantation across the septum, evacuation of the device within 28 days, and serious adverse events during the period of device intervention (up to 30 days). Symptomatic improvement (including Eckardt score [10]) was also monitored at 1 and 3 months.

\section{Results}

\section{Animal studies}

Cutting of the created gastric septum was confirmed within 1 week for pig \#1 (specimens explanted early for analysis [9]) and occurred between days 7 and 28 in pig \#2, after cutting of the septum was confirmed at autopsy. For pig \#3, dislodgement before cutting occurred within 24 hours. This was likely due to the fact that the double stomach thickness of the pig limited the force of the initial apposition of the magnets.

For pig \#4, migration of the device was confirmed after 14 days and the intestinal specimen was explanted. Macroscopically, the cutting of the artificially created septum was complete. Histological analysis showed that, after only 2 weeks, mucosa was growing on the cut site and that a full-layer healing scar was visible ( $\mathbf{F i g . 4 )}$.

\section{Clinical trial}

Two men of 73 and 56 years of age with a symptomatic EED were enrolled. From fluoroscopic images, the diverticulum and septum measured $52.5 \mathrm{~mm}$ and $25 \mathrm{~mm}$, respectively, in the first patient, and $58 \mathrm{~mm}$ and $40 \mathrm{~mm}$, respectively, in the second patient. Technical implantation was successful in both patients. Device insertion took 12 and 15 minutes for the first and sec- ond patients, respectively. No serious adverse events were reported.

For the first patient ( $\triangleright$ Fig.5), the MAGUS migrated spontaneously between days 14 and 28. The Eckardt score dropped from 2 to 1 between baseline and 28 days after the first intervention.

For the second patient, the cutting wire seemed to be blocked between the magnets. This happened because the distal magnet was still loosely attached to the catheter and was able to turn on itself, blocking the wire between the two magnets. Because the self-retraction of the wire was documented to be sufficient to induce its release, based on our previous experience, we decided to leave the device as it was. The magnets had not migrated after 28 days and endoscopy showed that the magnetic anastomosis was complete, but the wire was still blocked between the magnets. The wire was activated by pulling on it, and migration was confirmed after a further 12 days. The patient's Eckardt score dropped from 6 to 2 between baseline and 28 days after the first intervention.

\section{Discussion}

The results of this study show that the MAGUS device is a rapid, single-stage, and potentially safe and effective procedure that can be used to perform endoscopic sectioning of a septum in the $\mathrm{Gl}$ tract. The achievement of this during a single procedure has become feasible because of the addition of the self-retractable wire to the magnets. This wire applies a predefined range of pressure on the residual septum besides the position where the magnets are placed. The biological process following the localized ischemia induced by pressure necrosis is an inflammatory reaction that allows for progressive healing. This process is biological and not physical and does not occur in an explanted organ (data not shown). We have called this phenomenon "wire compression cutting".

EED is a rare condition that we selected for our first test in humans. The treatment of this entity has been evolving over the last few years. Although D-POEM seems to be a promising procedure compared with surgery, especially for EED associated with motility disorders like achalasia [2], the use of magnets 
has also been reported but requires a second step to cut the residual septum left after migration of the magnets $[3,4]$. This preliminary experience with a device that includes two magnets and a self-retractable wire shows that the endoscopist can successfully marsupialize an EED in an intended singlestep procedure.

The main advantage of this device is that it offers a less technically demanding procedure, based on the ease of use for the endoscopist and the application of the WCC concept. Nevertheless, the activation of the cutting wire required a second endoscopy in one patient. This problem can be solved at the initial insertion, if there is any doubt, by ensuring the immediate activation of the self-retractable wire by mobilizing it with a biopsy forceps.

Although the concept of the MAGUS device was originally tested in the setting of EED treatment, this limited human experience suggests that the safety profile of the procedure can allow us to consider other applications that may benefit from marsupialization. For example, Zenker diverticulum, candy cane syndrome (a postoperative complication of the afferent loop, for example post-bypass or after total gastrectomy) [11, $12]$, or anastomotic refractory strictures, in which a septum could be responsible for symptoms, represent potential and/or current areas of investigation.

Further clinical trials and follow-up are needed to assess the safety and efficacy of the treatment, as we have here presented only feasibility results. Clinical endpoints for further trials could focus on different adverse events, such as measurement of the risks of leakage, recurrence, or stenosis due to fibrotic scarring.

Further development of the device should focus on the improvement of the delivery catheter and procedure. This could ensure that the wire is properly activated at the end of the procedure and improve the learning curve of the procedure.

\section{Acknowledgments}

We would like to sincerely thank Innoviris.brussels (Brussels region), who financed this project. We would also like to acknowledge the contribution of medical writer Sandy Field, PhD for English language editing of this manuscript.

Funding

Innoviris Brussels RBC/2017-SOIB-9

\section{Clinical trial}

Trial Registration: ClinicalTrials.gov | Registration number (trial ID): NCT04480216 | Type of study: Feasibility and safety

\section{Competing interests}

Daniel Blero, Nicolas Cauche, Cécilia Delattre, Alain Delchambre, Jacques Deviere, Sonia Dugardeyn, François Huberland, and Ricardo Rio Tinto are the inventors of and holders of a patent on the MAGUS device. The remaining authors declare that they have no conflict of interest.

References

[1] Chan DSY, Foliaki A, Lewis WG et al. Systematic review and meta-analysis of surgical treatment of non-Zenker's oesophageal diverticula. J Gastrointest Surg 2017; 21: 1067-1075

[2] Yang J, Zeng X, Yuan X et al. An international study on the use of peroral endoscopic myotomy (POEM) in the management of esophageal diverticula: the first multicenter D-POEM experience. Endoscopy 2019; 51: 346-349

[3] Bouchard S, Huberty V, Blero D et al. Magnetic compression for treatment of large oesophageal diverticula: a new endoscopic approach for a risky surgical disease? Gut 2015; 64: 1678-1679

[4] Disibeyaz S, Saygili F, Oztas E et al. Endoscopic septotomy of a magnet-induced neoseptum in a large mid-esophageal diverticulum. Endoscopy 2016; 48: E244-E245

[5] Machytka E, Bužga M, Zonca P et al. Partial jejunal diversion using an incisionless magnetic anastomosis system: 1-year interim results in patients with obesity and diabetes. Gastrointest Endosc 2017; 86: 904-912

[6] Graves CE, Co C, Hsi RS et al. Magnetic compression anastomosis (Magnamosis): first-in-human trial. J Am Coll Surg 2017; 225: 676681.e1

[7] Zaritzky M, Ben R, Johnston K. Magnetic gastrointestinal anastomosis in pediatric patients. J Pediatr Surg 2014; 49: 1131-1137

[8] van Hooft JE, Vleggaar FP, Le Moine O et al. Endoscopic magnetic gastroenteric anastomosis for palliation of malignant gastric outlet obstruction: a prospective multicenter study. Gastrointest Endosc 2010; 72: 530-535

[9] Huberland F, Sandersen C, Bastin O et al. Wire and magnet septotome, a new method for endoscopic leak-free section of septa in the GI tract: preliminary result of a pilot animal study. Endoscopy 2019; 51: S172doi:10.1055/s-0039-1681679

[10] Eckardt VF, Aignherr C, Bernhard G. Predictors of outcome in patients with achalasia treated by pneumatic dilation. Gastroenterology 1992; 103: 1732-1738

[11] Aryaie AH, Fayezizadeh M, Wen Y et al. "Candy cane syndrome:” an underappreciated cause of abdominal pain and nausea after Roux-enY gastric bypass surgery. Surg Obes Relat Dis 2017; 13: 1501-1505

[12] Dallal RM, Cottam D. "Candy cane” Roux syndrome-a possible complication after gastric bypass surgery. Surg Obes Relat Dis 2007; 3 : 408-410 